\title{
La distribución del gasto educativo en la provincia de Buenos Aires, Argentina: ¿Es equitativa?
}

\author{
The distribution of educational expenditure in Buenos Aires province, Argentina: \\ Is it equitable?
}

\author{
María Marta Formichella ${ }^{1}$ \\ Universidad Nacional del Sur \\ Departamento. de Economía \\ Instituto de Investigaciones Económicas y Sociales del Sur \\ Consejo Nacional de Investigaciones Científicas y Técnicas \\ Bahía Blanca, Argentina \\ mfornichella@uns.edu.ar
}

Recibido: 26 febrero 2013 Aceptado: 4 diciembre 2014 Corregido: 16 diciembre 2014

\begin{abstract}
Resumen: La equidad en educación es socialmente deseable debido a la relevancia de la formación en el desarrollo de las personas. Por ello, resulta interesante estudiar si la asignación del gasto destinado a educación tiene en cuenta este aspecto. Dado que el concepto de equidad no es un unívoco, es necesario definir una postura; en el trabajo se asume que existe equidad educativa si hay igualdad en los resultados educativos. Así, el objetivo de éste es analizar si la distribución del gasto educativo entre los distritos de la provincia de Buenos Aires, Argentina, es equitativa. Se planteó la hipótesis de que no lo es. Para contrastarla, se utilizó el coeficiente de GINI y una regresión de Mínimos Cuadrados Ordinarios (MCO). Se halló que la distribución de los fondos se vincula en forma directa con el grado de analfabetismo de los distritos, pero al mismo tiempo se encontró evidencia de que es independiente de la pobreza, de la tasa de abandono escolar, del porcentaje de matrícula en los niveles iniciales y de la proporción de establecimientos clasificados como desfavorables. Entonces, se concluye que la distribución de los fondos de gastos educativos no se rige, o lo hace muy débilmente, por el principio de equidad considerado en el trabajo. Es decir, que la evidencia empírica verifica la hipótesis propuesta.
\end{abstract}

Palabras claves: equidad, educación, gasto.

Abstract: Equity in education is socially desirable because of the relevance of education for the development of people. Therefore, it is interesting to study whether the allocation of spending on education takes into account this aspect. Since the concept of fairness is not univocal, it is necessary to define a position. Here, it is assumed that there is educational equity if equality exists in educational

1 Doctora en Economía de la Universidad Nacional del Sur. Actualmente trabaja como investigadora del Concejo Nacional de Investigaciones Científicas y Técnicas (CONICET), y como docente de la Cátedra "Indicadores Económicos y Cuentas Nacionales" de la Universidad Nacional del Sur (UNS). Anteriormente trabajaba en el Instituto Nacional de Tecnología Agropecuaria (INTA). Ha participado como ponente en numerosos congresos nacionales e internacionales. Entre sus recientes publicaciones se destacan: Equidad educativa en Argentina: Análisis desde la perspectiva de los resultados educativos en Investigaciones de Economía de la Educación; ¿Se debe el mayor rendimiento de las escuelas de gestión privada en la Argentina al tipo de administración? en Revista de la CEPAL; Análisis del concepto de equidad educativa a la luz del enfoque de las capacidades de Amartya Sen en Revista Educación 35;Reflexiones sobre el rol de la educación universitaria en el desarrollo en Revista Latinoamericana de Estudios Educativos, entre otras. 
outcomes. Thus, the purpose of the paper is to examine whether the distribution of educational spending among the districts of the Province of Buenos Aires, Argentina, is equitable. The hypothesis that it is not, is proposed. The Gini coefficient and an Ordinary Least Squares $(O L S)$ regression are used to verify this hypothesis. It was found that the distribution of funds depends directly on the degree of illiteracy among districts, but also there is evidence that is independent of poverty, dropout rate, percentage of enrollment in the initial levels and the proportion of establishments classified as unfavorable. Then, it is concluded that the educational expenses distribution is not governed, or it is only done very weakly, by the principle of equity in the paper considered. That is, empirical evidence verifies the proposed hypothesis.

Keywords: equity, education, expenditure.

\section{Introducción}

Diversos autores dentro de la literatura económica han estudiado la relación entre la educación y el crecimiento del producto y han hallado una vinculación positiva entre estas variables (Barro y Lee, 2013; Lucas, 1988; Nelson y Phelps 1966, en Aghion y Howitt 1998). En este sentido, algunos han destacado el rol de la distribución de la educación como determinante del progreso económico (Castello y Doménech, 2008; Thomas, Wang y Fan, 2002; López, Thomas y Wang, 1998; Birdsall y Lodoño, 1997).

Asimismo, la búsqueda de la equidad en educación es un objetivo social considerado deseable (Formichella, 2010; Llach, 2006; Morduchowicz, 2003). Algunos autores argumentan esta postura a partir de reconocer el rol de la educación en el desarrollo de las personas (Formichella, 2010; Santos, 2007; Thomas et. al., 2002, Sen, 1999); otros resaltan que la equidad educativa aumenta la equidad económica (Guadagni, 2007; Gasparini, 2002). Por último, hay un grupo de autores que postulan que las oportunidades de las personas (entre ellas su posibilidad de educarse) deben ser igualadas con el fin de construir una organización social justa (Ribotta, 2006; Sen, 1999; Roemer, 1995, Cohen, 1989 ; Arneson, 1989, en Ribotta, 2006).

El objetivo del presente trabajo es analizar si la distribución del gasto educativo desde el gobierno central de la provincia de Buenos Aires, hacia los diferentes distritos escolares pertenecientes a dicho territorio, es realizada bajo una lógica de equidad. La provincia de Buenos Aires se encuentra situada en el centro de la República Argentina; es la más poblada y representa el 39\% de los habitantes en suelo argentino.

Es importante hacer tres aclaraciones: a) desde la década de 1990 el financiamiento de la educación en Argentina se encuentra prácticamente 100\% en manos de las provincias (Rojas y Formichella, 2011), de allí que sea más pertinente realizar un análisis por provincia, que a nivel país; b) si bien el interés del trabajo se centra en Buenos Aires, la metodología podría replicarse para analizar la cuestión en otras provincias de la Argentina o en otros países; y c) el objetivo planteado se vincula a la idea de equidad educativa; sin embargo, éste no es un concepto unívoco, al igual que el concepto de equidad social. Por ello, en el trabajo se discute esta acepción y se opta por una definición alternativa.

La cantidad de fondos que recibe cada uno de los distritos de la provincia de Buenos Aires se precisa con base en la Ley Provincial $\mathrm{N}^{\circ} 13010$, donde se establece que el $25 \%$ de lo recaudado por medio del impuesto a los Ingresos brutos será destinado al mantenimiento de Establecimientos Educativos, a través de un fondo compensador, el cual será distribuido 
entre los distritos de acuerdo con lo que decida la Dirección General de Cultura y Educación de la Provincia. Entonces, la pregunta a responder en esta investigación es la siguiente: ¿esas decisiones se basan en criterios de equidad? Como respuesta al interrogante expresado, en el trabajo se propone la hipótesis de que esto no ocurre. Es decir, se postula que la asignación del gasto educativo en la provincia de Buenos Aires no es equitativa.

A continuación, se detallan algunos antecedentes sobre el tema, luego, se presenta el marco teórico y, posteriormente, se explica la metodología empleada. En la sección cinco se describen los datos y las variables utilizadas; en la seis se presentan los resultados encontrados del estudio y, finalmente, en la siete se exponen las conclusiones.

\section{Antecedentes}

Existen diversas maneras de abordar el tema de la equidad educativa y su relación con el gasto destinado a la prestación de los servicios educativos. Algunos autores estudian en qué medida son las familias las que hacen aportes económicos en dicho sentido y en qué proporción la contribución proviene del gasto público (esta arista se vincula directamente con los análisis referidos a la provisión pública y privada de los servicios educativos). Otros autores se concentran en qué porción del gasto público se destina a educación y cuáles son los sectores sociales que resultan beneficiados. Asimismo, hay quienes focalizan su atención en la distribución del gasto público por finalidad y función, para ver en detalle qué proporción de éste se corresponde con el gasto educativo. Finalmente, cabe mencionar que hay autores que analizan cómo se distribuye geográficamente el gasto educativo (Morduchowicz, 2003).

En el último caso citado, las transferencias desde un gobierno central hacia entidades geográficas menores pueden tener un impacto redistributivo. Para que así sea, el monto de la transferencia debe ser inversamente proporcional al nivel de renta per cápita (De Mello e Sousa, 2006).

Esta idea coincide con el planteo de De La Torre (2004), quien estudia si el gasto público destinado a desarrollo (el cual incluye el gasto en educación), se asigna entre las entidades federativas de México en forma proporcional e inversa al Índice de Desarrollo Humano (IDH) de cada una de éstas. De la Torre (2006, p.6) expresa que de esa manera se podría colaborar con la mejora de las zonas más rezagadas en salud, educación e ingresos (dimensiones que considera el IDH); sin embargo, en su análisis empírico encuentra que esto no se verifica.

Cabe señalar que De la Torre (2004) no es el único autor que detecta la existencia de mecanismos que no contribuyen con la equidad educativa entre regiones geográficas, sino que existen investigadores que, mediante sus trabajos, presentan ideas vinculadas con dichos mecanismos. Entre ellos, pueden mencionarse Ontiveros Jimenez (2001, 2003), Baaldante y Geldres (2003), Jaramillo y Artiga (2004), Rivas y Mezzadra (2005), Nina, Molina, Barrientos y Aguilar (2006), Sanchez (2006), Neyra Aráoz (2010), Alas Solis y Moncada Godoy (2010), Rojas y Formichella (2011) y Gil Flores (2014).

Ontivero Jiménez $(2001,2003)$ analiza los determinantes de la distribución del gasto educativo entre los diferentes estados de México. Halla que el principal criterio que rige la asignación del gasto es el número de maestras y maestros contratados en cada estado. Asimismo, no encuentra que exista una mayor transferencia de recursos hacia los estados más pobres o de menor nivel educativo. Por último, recoge evidencia que indica que los estados 
con mayores tasas de alfabetización dedican más recursos propios a la educación que aquellos cuya población posee mayores problemas de analfabetismo.

Baaldante y Geldres (2003) estudian cuáles son los principales criterios que explican cómo se distribuye el gasto educativo entre Unidades Ejecutoras en Perú. Definen las unidades ejecutoras como aquellas entidades autorizadas por el Ministerio de Economía, perteneciente al gobierno central, para ejecutar presupuesto (por ejemplo: una Dirección Regional o Direcciones Sub Regionales de Educación, o alguna Unidad de Servicios Educativos o Área de Desarrollo Educativo). Estos autores encuentran que las variables institucionales, específicamente las organizacionales, son las más relevantes a la hora de explicar la asignación del gasto. Por otra parte, no hallan señales de que exista influencia de maestros o padres de familia, quiénes constituyen los principales grupos de interés. Finalmente, respecto a la existencia de mecanismos redistributivos vinculados a las características socio-demográficas, sugieren una distribución regresiva del gasto por alumno; aunque al mismo tiempo encuentran que las transferencias destinadas a áreas geográficas, con mayor tasa de analfabetismo y mayor presencia de población originaria, son proporcionalmente superiores.

Jaramillo y Arteaga (2004) también analizan la distribución del gasto educativo entre Unidades Ejecutoras en Perú. Observan que la asignación del gasto total por estudiante no depende del nivel de pobreza y, a diferencia de los autores citados previamente, tampoco hallan vinculación con los indicadores de carencia educativa (como la tasa de analfabetismo o la proporción de población de pueblos originarios). Esta evidencia sugiere que la política del gasto educativo no atiende a principios de equidad.

Rivas y Mezzadra (2005) y Rojas y Formichella (2011) estudian el caso de Argentina y proponen el debate acerca de la actual distribución de ingresos entre las provincias. Muestran que dicha distribución no se realiza con base en objetivos de justicia social, lo cual implica que existan amplias desigualdades educativas entre ellas.

Nina et. al. (2006) analizan la equidad educativa entre regiones geográficas en Bolivia y encuentran que sería necesaria una redistribución en pos de mejorarla. Los resultados de su análisis empírico sugieren que se deberían aumentar las transferencias hacia las áreas con mayores necesidades educativas y peor rendimiento académico. Estos autores explican que de ese modo se podrían limitar las influencias negativas que tienen ciertos contextos, como la pobreza y el bajo nivel educativo de los padres, sobre los resultados escolares del alumnado.

Por su parte, Sánchez (2006) estudia el caso de Colombia y halla que las transferencias destinadas a los municipios para la prestación de los servicios educativos tienen un impacto negativo en la tasa de escolarización, mientras que los esfuerzos propios evidencian un efecto positivo. Este resultado sugiere que dichas transferencias no son inversas al grado de desarrollo y, por ende, no representan un mecanismo de redistribución.

Neyra Aráoz (2010) también investiga sobre la equidad educativa en Perú. Presenta evidencia acerca de la existencia de mecanismos fiscales que profundizan las desigualdades educativas regionales. Asimismo, propone un sistema redistributivo que favorezca a aquellas regiones más desfavorecidas en términos de pobreza y más atrasadas en cuanto a logros escolares.

Alas Solis y Moncada Godoy (2010) analizan la equidad educativa en Honduras. Encuentran diferentes focos de desigualdad, entre ellos, evidencian una gran brecha entre 
regiones, sobre todo entre el ámbito urbano y rural. Concluyen que el gasto público destinado a educación debería focalizarse en las zonas más desfavorecidas y destinarse en mayor proporción a éstas.

Por último, y bajo la aclaración de que esta enumeración de estudios de investigación no ha sido taxativa, cabe mencionar el trabajo de Gil Flores (2014). Este autor analiza las desigualdades educativas entre las Comunidades Autónomas en España. Encuentra que la población de la región norte, con mayor nivel de producto y empleo, y menores problemas de pobreza, obtiene resultados educativos más elevados que la correspondiente a la región sur, cuyas características socioeconómicas son inversas a las descritas. Concluye que los factores de contexto son los que más influyen sobre el desempeño educativo, dándole menos importancia a la disponibilidad de recursos y mayor relevancia a cómo éstos se utilizan.

\section{Marco Teórico}

Respecto a los determinantes del gasto educativo, Baaldante y Geldres (2003) explican que la literatura propone tres enfoques: a) una perspectiva de demanda que los estudia de acuerdo con la mirada de los entes consumidores (familias, alumnos, comunidad en general, etc.) y centra la importancia en las variables socioeconómicas y demográficas; b) un análisis desde el punto de vista de la oferta, que se centra en el rol de los grupos de interés y en qué influencia pueden tener en las decisiones de quienes determinan el presupuesto; y c) un enfoque que se detiene en la equidad en la asignación del gasto y estudia qué elementos afectan su distribución, teniendo en cuenta tanto factores sociales, demográficos y económicos como políticos (vinculados a los grupos de interés).

Por otra parte, Molina Morales, Amate y Guarnido (2011) clasifican los determinantes del gasto en tres grupos: sociales y demográficos, políticos e institucionales. El primero se relaciona con el enfoque de demanda y el segundo, con el de oferta. El tercero tiene que ver con el tipo de organización fiscal; es decir, si se trata de un sistema educativo centralizado o descentralizado para ese efecto.

El presente trabajo se enmarca dentro del tercer enfoque explicado por Baaldante y Geldres (2003). Por ello, se requiere de una definición del concepto de equidad para luego especificar si la distribución del gasto en determinado contexto geográfico se orienta o no a la consecución de ésta.

Si bien la palabra equidad proviene del latín (aequitas) y significa igual, en función de cuál sea la base de información que se utilice para igualar a las personas, existen diferentes acepciones del concepto (Sen, 1979). Entonces, equidad significa igualdad entre individuos en algún atributo y en educación implica igualdad en algún aspecto referido al alumnado.

Marc Demeuse (en López, 2006) explica que existen cuatro principios de equidad en educación: a) igualdad en el acceso, b) igualdad en las condiciones de aprendizaje, c) igualdad en los resultados e d) igualdad en la realización social de dichos resultados.

El primer criterio es una especie de igualdad de oportunidades basada en los recursos escolares y representa una propuesta que avala la instalación de un sistema meritocrático. Esto 
significa que lo que cada individuo logra depende únicamente de su propia responsabilidad, esfuerzo y talento (López, 2006). Un sistema educativo basado en la meritocracia profundizaría en las diferencias de origen y legitimaría el fracaso escolar. Por ello, no será el enfoque de equidad que se considere en este trabajo.

Con respecto al segundo punto de vista, se hace referencia a la igualdad en los procesos de enseñanza. Esto significa tratar de la misma manera a todas las personas que ingresan en el sistema educativo, es decir, utilizar las mismas estrategias (López, 2006). Este principio no tiene en cuenta la educabilidad de los individuos y por ello tampoco será el criterio considerado en el presente trabajo. Tedesco (2000) define la educabilidad como aquellas condiciones sociales necesarias para que un individuo pueda ir a la escuela y tener éxito. Éstas tienen que ver con aspectos de desarrollo que se vinculan con el contexto más cercano del alumnado, ya que incluyen la estimulación afectiva, la alimentación, las condiciones sanitarias y la socialización primaria.

En relación con el criterio basado en la igualdad en la realización social de los logros, la equidad es evaluada en función del impacto que tiene un sistema educativo en el desarrollo social. En este caso, la educación es valorada en términos del desarrollo regional que pueda aportar y no como un bien en sí mismo (López, 2006). Por tal motivo, tampoco es el enfoque de equidad aquí escogido.

Por último, resta analizar el principio de equidad basado en la igualdad en los resultados. Morduchowicz (2003) explica que éste no contradice ni es incompatible con la igualdad en las oportunidades, sino que implica que se establezcan las condiciones y se comprometan los recursos necesarios para que todos los estudiantes tengan la misma oportunidad de arribar a los resultados establecidos como deseables. Este autor expone claramente que para alcanzar resultados homogéneos es imprescindible actuar de manera heterogénea.

Para que todos los individuos egresen del sistema educativo habiendo alcanzado un mismo resultado, es imprescindible que accedan a él y, una vez allí, sus diferencias de origen puedan ser compensadas. Es decir, que cada uno pueda disponer de los recursos materiales (aulas, bancos, etc.) e inmateriales (tiempo, apoyo psicológico, enseñanza, motivaciones, entre otros) requeridos para transitar con éxito su carrera escolar. Esto implicaría que no todos reciban lo mismo, ya que algunos necesitarán más que otros. Aquí es relevante hacer una aclaración: es de esperar que el ámbito educativo no sea suficiente para compensar las diferencias de origen de las personas y cuanto mayor sea el grado de desigualdad inicial, más limitada se encontrará la institución escolar para hacer frente a las compensaciones. Esto significa que, probablemente, sea necesaria la aplicación de políticas vinculadas a otros ámbitos para poder obtener el resultado deseado (Formichella, 2010). Entonces, si lo que se busca es la igualdad en los resultados educativos, es necesario aplicar dos principios postulados por Aristóteles: "Igual trato para los iguales" (equidad horizontal) y "desigual trato para los desiguales" (equidad vertical) (Morduchowicz, 2003).

En igual sentido, Sen $(1979,1999)$ explica que la noción de equidad conlleva implícito que, para lograr la igualdad en un atributo, puede ser necesario instaurar desigualdades en otros. En educación, esto implica que se deberían tomar en cuenta las diferencias de origen y las individualidades de cada estudiante, lo que haría necesario abogar por algunas desigualdades en la trayectoria educativa del alumnado (Llach, 2006; Tedesco, 2000). El último criterio 
enunciado, al buscar que todos los individuos accedan a cierto grado de educación, la valora como un bien en sí misma. Además, es un punto de vista completo e integral, ya que incluye oportunidades, procesos y resultados. Por ello, es el enfoque escogido en este estudio.

En suma, en educación, dado que los individuos parten de condiciones heterogéneas, no todos lograrían alcanzar los mismos resultados aún si se enfrentaran a idénticas condiciones en el ámbito escolar; por ende, una situación de equidad, en el sentido aquí propuesto, implica que se compensen las diferencias de origen, dando lugar a la igualdad en los logros educativos finales (Formichella, 2010).

Queda fuera del alcance de este trabajo discutir qué resultados homogéneos podría buscar alcanzar el sistema educativo, puesto que es un debate que cada sociedad debería procurarse $^{2}$. Sin embargo, es preciso mencionar que la igualdad en resultados tiene que basarse en un mínimo común, ya que la igualdad absoluta no podría evidenciarse debido a las diferencias naturales existentes entre los individuos (Formichella, 2010).

Finalmente, cabe mencionar que, a partir del análisis referido a los parámetros que guían la distribución del gasto educativo, es posible determinar si esa asignación es realizada o no con algún objetivo de equidad y, de ser así, bajo cuál criterio. Por ejemplo, una distribución igualitaria del gasto por alumno refleja una visión de la equidad basada en el acceso; mientras que una distribución desigual y progresiva de éste -en el sentido de que reciban más recursos los que poseen mayores carencias de origen-, refleja una postura de equidad basada en los resultados educativos.

\section{Metodología ${ }^{3}$}

Siguiendo a Ontiveros Jiménez (2003), que analiza la distribución del gasto educativo entre los diferentes estados de México, se utiliza como herramienta de análisis la metodología econométrica de Mínimos Cuadrados Ordinarios (MCO). El software elegido para trabajar es el STATA 11.1. En un modelo de Mínimos Cuadrados Ordinarios se representan las relaciones entre una variable dependiente (denominada Y) y una o más variables independientes (denominadas $\mathrm{X}$ ), de manera lineal. Además, se supone que hay un término independiente $\left(B_{0}\right)$ y un término que representa la existencia de cierto grado de error aleatorio ( $\mu \mathrm{i})$. A su vez, se supone que este tiene media cero y varianza constante. La expresión formal es la que se muestra en la ecuación (1):

$$
\mathrm{Y}_{\mathrm{i}}=B_{0}+B_{1}^{*} \mathrm{X}_{1 \mathrm{i}}+B_{2} * \mathrm{X}_{2 \mathrm{i}}+\ldots \ldots+B_{\mathrm{n}}^{*} \mathrm{X}_{\mathrm{ni}}+\mu_{\mathrm{i}}
$$

Lo que representa la expresión anterior es una regresión múltiple que permite controlar simultáneamente muchos factores que influyen en una variable. La variable Y está

2 Para un mayor análisis al respecto ver Formichella (2010).

3 Este apartado se basa en el libro "Introducción a la econometría: Un enfoque moderno" (Wooldridge, J. 2001). Aquí se explican brevemente los conceptos básicos de la metodología y las herramientas necesarias para comprender la interpretación de los resultados. Por lo tanto, se recomienda que el lector que desee mayores detalles sobre estos temas consulte dicho libro. 
influida por las variables "X" y por variables inobservables que se encuentran contenidas en "u". La metodología de MCO permite estimar en qué medida afecta cada X a la variable dependiente, manteniendo el resto de los factores constantes (ceteris paribus). Es decir, por ejemplo, " $\mathrm{B}_{1}$ "mide el cambio en "Y" con respecto a " $\mathrm{X}_{1}$ ", manteniendo los demás factores fijos. Habitualmente, una o más variables incorporadas en la regresión tienen la finalidad de dar respuesta a alguna pregunta planteada como hipótesis acerca de la Y, mientras que otro grupo de variables son incorporadas al modelo como control. Es decir, con el fin de analizar el efecto neto de las " $\mathrm{X}$ " de interés y extraer conclusiones sin que dicho efecto se encuentre sesgado por la influencia de otras variables.

El objetivo del modelo es estimar los valores de los parámetros B. El método de Mínimos Cuadrados Ordinarios escoge aquellos estimadores que hagan mínima la suma de los cuadrados de los residuos. Esto quiere decir, que dada cierta cantidad de observaciones (N) de la "Y" y de las "X", el método elige los estimadores $B$ (los señalamos con ${ }^{\wedge}$ ) que hacen que la diferencia entre el valor verdadero de la variable de interés "Y" y su valor estimado sea lo más pequeña posible. Es decir, elige los estimadores que minimizan la siguiente diferencia, representada en la ecuación (2):

$$
\sum_{1}^{n}\left(Y_{i}-\widetilde{\beta_{0}}-\widetilde{\beta_{1}} X_{i 1}-\widetilde{\beta_{2}} X_{i 2 . . .} \widetilde{\beta_{n}} X_{i n}\right)^{2}
$$

Finalmente, el valor de cada parámetro " $B$ " estimado representa en qué medida la "X" que lo acompaña influye, ceteris paribus, en el valor de la variable dependiente "Y".

Cabe señalar que, al proponerse un modelo de MCO para explicar la variable de interés (Y), no necesariamente el modelo será útil para llevar a cabo dicha explicación ni todas las variables resultarán ser individualmente relevantes para el logro del mencionado objetivo. Dado que es de interés plantear un modelo que resulte explicativo y detectar qué variables son importantes, se realizan pruebas de hipótesis estadísticas para dar respuesta a estas inquietudes.

Con el fin de conocer si todas las variables, en forma simultánea, resultan explicativas de la variable dependiente, se realiza una prueba de significatividad conjunta (comúnmente denominada "Prueba F"). La hipótesis nula es que todos los coeficientes (los "B") son iguales a cero y, por lo tanto, las variables independientes consideradas no son útiles para explicar el comportamiento de la dependiente. La hipótesis alternativa es la contraria, es decir que son diferentes a cero y útiles para caracterizar la "Y". Se utiliza un estadístico F de Fisher-Snedecor para realizar la prueba y determinar si la hipótesis nula se rechaza o no. El software usado en este trabajo (STATA 11.1) permite llevar adelante esta prueba y reporta el nivel de significancia estadística más pequeño al que se rechazaría la hipótesis nula (denominado p-value).

Se acepta decir que las variables son estadísticamente significativas al explicar la variable dependiente, si el p-value es menor o igual a 0,10. Esto implica que la hipótesis nula se rechaza al 10\%, es decir que la probabilidad de cometer el error de rechazar la hipótesis nula y que ésta sea verdadera (denominado error tipo I) es de 0,10 y se le otorga un nivel de confianza del $90 \%$ a la respuesta. El nivel de confianza es justamente el grado de tolerancia a 
la posibilidad de cometer el error tipo I. Cuanto menor sea el p-value, menos probable es que se cometa este error y más certero es el resultado.

Si bien a partir de la prueba de significatividad conjunta es posible afirmar que las variables "Xs" explican en forma simultánea a la variable "Y", existe un indicador de la bondad de ajuste del modelo, comúnmente utilizado y denominado $\mathrm{R}^{2}$. Éste "representa la proporción de la variación explicada en la variación total y, por tanto, se interpreta como la fracción de la variación muestral de Y que es explicada por las Xs" (Wooldridge, 2001, p. 39).

Con el objeto de conocer qué variables independientes resultan estadísticamente significativas en forma individual a la hora de explicar la variable dependiente, se pueden realizar pruebas de significatividad individual sobre cada coeficiente "B" (comúnmente denominada "Prueba t"). La hipótesis nula es que el coeficiente en cuestión es igual a cero y, por lo tanto, la variable "X" que lo acompaña no es relevante para explicar la variabilidad de la variable dependiente "Y". La hipótesis alternativa es la contraria, es decir que el coeficiente en cuestión es diferente de cero y la variable "X" que le corresponde es útil para explicar el comportamiento de la "Y". Se utiliza un estadístico t de Student para realizar la prueba y determinar si la hipótesis nula se rechaza o no.

El software usado en este trabajo (STATA 11.1) permite llevar adelante la prueba descrita y reporta el nivel de significancia estadística más pequeño al que se rechazaría la hipótesis nula (denominado p-value). Al igual que en el caso de la prueba de significatividad conjunta, suele ser aceptable decir que una variable " $X$ " es estadísticamente significativa para explicar la variación de la variable dependiente "Y" si el p-value es menor o igual a 0,10 . Esto quiere decir que la hipótesis nula se rechaza al 10\%, es decir que la probabilidad de cometer el error de rechazar la hipótesis nula y que ésta sea verdadera (el cual, como ya se ha mencionado, es denominado error tipo I) es de 0,10 lo cual otorga un nivel de confianza del $90 \%$ a la respuesta.

Por último, cabe señalar que una variable " $\mathrm{X}$ " puede resultar estadísticamente significativa para explicar la variación de la dependiente "Y", pero no ser relevante en función del problema bajo estudio. Es decir, puede ser que el valor del coeficiente que acompaña a la "X" sea muy bajo en relación con fenómeno que se analiza y que esto la haga poco importante o inútil para explicar la variabilidad de "Y".

Por otra parte, es necesario exponer la metodología de cálculo del denominado Coeficiente de GINI, ya que es el instrumento utilizado para calcular el grado de desigualdad existente en la asignación de gasto educativo entre los distritos de la provincia de Buenos Aires.

El coeficiente de GINI ha sido ampliamente utilizado como medida de distribución del ingreso. Éste es atribuido a Gini (1912) y estudiado luego por diversos autores, tales como Ricci (1916) y Atkinson (1970), entre otros (Sen, 1997). Si bien este coeficiente es habitualmente utilizado para medir la dispersión en los ingresos personales de los individuos, también suele usarse como medida de desigualdad en la distribución de variables vinculadas a la educación, tales como el número de años de estudio aprobados o el gasto educativo, entre otras (Formichella, 2010).

Este coeficiente se forma a partir de la curva de Lorenz. La curva de Lorenz surge de graficar la distribución acumulada de observaciones ordenadas según determinada variable 
de interés (por ejemplo: gasto educativo), en el eje de las X, contra la distribución acumulada de dicha variable en el eje de las Y (Morduchowicz, 2003). Por ejemplo: si se elige utilizar la variable "gasto educativo asignado por distrito", en el eje X se ordenan los distritos según la asignación de gasto educativo que obtiene cada uno y en el eje $\mathrm{Y}$ se acumula el gasto educativo. En el total acumulado, para el 100\% de distritos ordenados según la asignación de gasto recibida, se tendrá el total de fondos acumulados por la población objetivo. Por ello, la curva termina en la esquina superior del cuadrado y la diagonal representa una situación de perfecta igualdad (Lugo, 2005; Calero, Escardibul y Mediavilla, 2006).

Es decir, cuando esta curva es igual a la diagonal existe perfecta igualdad; de lo contrario, la desigualdad es mayor cuanto más se aleje de ésta. Precisamente, el Coeficiente de Gini mide el área que se encuentra entre las dos curvas; varía entre cero y uno y la distribución es más igualitaria cuánto más se acerque a cero (Morduchowicz, 2003).

Por ello, el coeficiente de Gini se define como la razón de la diferencia entre estas curvas y la región triangular que se encuentra debajo de la diagonal (Sen, 1997). Thomas, Wang y Fan (2001) presentan la siguiente expresión para calcular el coeficiente de Gini:

$$
G=\frac{1}{\mu N(N-1)} \sum_{i>j} \sum_{j}\left|e_{i}-e_{j}\right|
$$

Donde:

- $\mu$ es el promedio de la variable educativa en cuestión.

- $\quad N$ es el número total de observaciones

- $e_{i}$ y erepresentan de los valores de la variable educativa de la observación $i$ y $j$.

\section{Los datos y las variables utilizadas}

Respecto de los datos, se utilizó una base que fue construida especialmente para llevar a cabo esta investigación. En ella, se recopilaron datos de 2009, debido a que es el año más actual del cual se cuenta con información sobre todas las variables de interés.

La información acerca del financiamiento del Consejo Escolar ${ }^{4}$ de cada distrito y de la clasificación de los establecimientos, de acuerdo con las condiciones desfavorables, se obtuvo de la página Web de la Dirección General de Cultura y Educación de la Provincia de Buenos Aires. Los datos vinculados a la matrícula fueron publicados en el relevamiento anual 2009, llevado a cabo por la Dirección Nacional de Información y Evaluación de la Calidad Educativa (DINIECE); mientras que los datos relacionados a características estructurales de los distritos se obtuvieron del Censo Nacional de Población y Vivienda del año 2010. De este modo, se construyó una base de datos con 114 observaciones correspondientes a 114 de los 134

$4 \quad$ Ente encargado de ejecutar el presupuesto educativo en cada distrito. 
distritos de la Provincia de Buenos Aires.

Como se mencionó, la regresión de MCO se llevó a cabo a través del software STATA 11.1. Se tomó como variable dependiente el "monto de dinero que el gobierno central de la provincia le otorga a cada distrito para afrontar los gastos en insumos escolares y el mantenimiento de la infraestructura del sector educativo". Es decir, en esta variable no se incluye el pago de salarios docentes ni el gasto destinado al Servicio Alimentario Escolar (SAE).

El gasto en salarios no se incorporó al análisis porque no se distribuye a través de los Consejos Escolares. El gasto destinado a SAE se excluyó porque, si bien abastece de recursos a los comedores escolares y es necesario para que el alumnado estudie, no se trata de un gasto educativo propiamente dicho. Este es un gasto que suple la falta de alimentación que el estudiantado debería recibir en su hogar, que es relevante más allá del rol que cumplen en la escuela.

La variable descrita párrafos atrás se denominó "Total de Fondos Provinciales en Infraestructura e Insumos educativos"(TFPII)y se incorporó al modelo comoTFPIIi;es decir, como "Total de Fondos Provinciales en Infraestructura e Insumos educativos recibidos por el distrito i".

Las variables independientes que se usaron fueron las siguientes:

- Porcentaje de establecimientos en condiciones desfavorables(ED): indica qué porcentaje de establecimientos escolares cumplen con la característica de ser codificados como "desfavorables". Esta es una de las clasificaciones de los establecimientos de enseñanza en función de su ubicación y/o dificultades de acceso, de acuerdo a criterios establecidos por la Dirección General de Cultura de Educación. Tanto la ubicación como las dificultades de acceso generan condiciones de trabajo diferenciales para docentes y estudiantes (Dirección General de Cultura y Educación, 2012). Si la distribución de fondos se rige por el criterio de equidad asumido en este trabajo, la mencionada variable tendrá signo positivo, es decir que los distritos con mayor proporción de establecimientos desfavorables reciben una mayor cantidad de fondos; en cambio, si esta variable resulta ser no significativa o su signo negativo, esto representaría evidencia a favor de la hipótesis.

- Tasa de abandono (TA): se define como la variación porcentual entre la matricula final e inicial en 2008 (año anterior al correspondiente en la base de datos). En un contexto de búsqueda de equidad, se esperaría que tenga signo positivo; es decir, que los distritos que presentan mayores dificultades para retener a sus estudiantes reciban más financiamiento. Por el contrario, en caso de que la variable sea significativa y su signo negativo, o en caso de que resultara no ser significativa, ello sería evidencia a favor de la hipótesis de este trabajo.

- Necesidades Básicas Insatisfechas (NBI): Dado que la información correspondiente a esta variable surge de datos de los censos de población y vivienda, y considerando que aún no se encuentra disponible para el último censo realizado en la Argentina (Censo Nacional de Población y Vivienda 2010), se utiliza, como aproximación, el porcentaje de hogares que no posee baño o cuyo baño es precario. En un contexto de política educativa progresiva, se esperaría que esta variable tenga signo positivo, lo cual significaría que los distritos que poseen una mayor proporción de población con 
dificultades en el acceso a los recursos para satisfacer sus necesidades, recibirían un financiamiento mayor para poder hacer frente a los requerimientos de compensación. En cambio, si la variable resultara no ser significativa o tuviera el signo negativo, se confirmaría la hipótesis propuesta.

- Analfabetos (A): Esta variable se define como el porcentaje de la población mayor a 10 años que no sabe leer y escribir. Si la variable resulta ser significativa y su signo positivo, implicaría que existe una política tendiente a otorgar mayor financiamiento a los distritos que más afectados están por este problema. Por el contrario, si su signo fuera negativo o no fuera significativa, se estaría aportando evidencia a favor de la hipótesis presentada.

- Porcentaje de alumnos que asisten a los niveles inicial y primario (AIP): Indica qué proporción de la matrícula se encuentra cursando los primeros niveles. En un contexto de búsqueda de equidad, esta variable tendría signo positivo, ya que, por lo explicado anteriormente, el primer paso para compensar las diferencias de origen es que el alumnado reciba dicha compensación en los primeros años escolares. Esto con el fin de que tenga la oportunidad de equiparar al resto (recordar que se mencionó la meta de equiparación haciendo referencia a la igualdad en los resultados mínimos, ya que la igualación total es imposible en un contexto de diferencias individuales innatas y naturales). En caso de que esta variable sea significativa y su signo negativo, o en caso de que resultara no significativa, se estaría avalando la hipótesis presentada en este estudio.

- Matrícula (M): Indica la cantidad de estudiantes de cada distrito que asiste al sector educativo de gestión estatal e incluye todos los niveles educativos presentes. Dado que la matrícula representa al estudiantado registrado en una unidad educativa, a una fecha determinada, de acuerdo con las normas pedagógicas y administrativas vigentes, y varía respecto del momento en que se efectúe el recuento (Dirección Provincial de Información y Planeamiento Educativo de la Provincia de Buenos Aires, 2012), cabe señalar que aquí se utiliza como dato la matrícula al 30 de abril de 2009. Se introduce como variable de control, justamente para captar el efecto causado por las diferencias de tamaño entre los distritos. Se espera que esta variable tenga un signo positivo; es decir, que los distritos que poseen una mayor cantidad de estudiantes reciban un mayor financiamiento.

- Partido Político (PP): Es una variable dicotómica que toma valor 1 si el intendente del distrito pertenece al mismo partido político que el gobernador en el año considerado y su valor es cero 0 si pertenece a otro partido. Se utiliza como variable de control con la finalidad de captar si la coincidencia política genera algún sesgo en la distribución de los fondos.

- Unidades educativas por establecimiento (UEPE): indica la cantidad de Unidades educativas que, en promedio, se encuentran en funcionamiento por establecimiento ${ }^{5}$. Se introduce como variable de control y se espera que tenga signo positivo, ya que cuantas más unidades educativas haya en funcionamiento en un establecimiento,

5 "Una Unidad Educativa (UE) es la unidad organizacional conformada al interior de un establecimiento educativo que tiene por objetivo impartir educación en torno a un tipo de educación (común, especial, artística, adultos) y a un determinado nivel de enseñanza (inicial, EGB 1 y 2, EGB 3, polimodal, superior no universitario). Este concepto se distingue del de establecimiento, porque un establecimiento tendrá tantas unidades educativas como niveles y tipos de educación ofrezca” (Dirección General de Cultura y Educación, 2012). 
mayor es la presión sobre los recursos y mayor la necesidad de financiamiento; por ende, se espera que los distritos que poseen mayor concentración de unidades educativas en sus establecimientos reciban una mayor cantidad de recursos para hacer frente al desgaste de infraestructura.

- Fondos propios (FP): indica los fondos propios que cada distrito posee y destina a educación. Se introduce como variable de control y se espera que tenga un signo positivo; es decir, que los distritos que poseen mayores recursos -una mayor capacidad económica- reciban menos por parte de la provincia.

\section{Resultados}

Con el fin de probar la hipótesis propuesta, se hace uso de dos herramientas: el coeficiente de GINI y una regresión econométrica de Mínimos Cuadrados Ordinarios. En primer lugar, utilizando el Software STATA 11.1 se calcula el Coeficiente de GINI para la variable definida como "Fondos Provinciales en Infraestructura e Insumos por alumno" (FPIIPA).

Berne y Stiefel (1994, citados por Morduchowicz, 2003) destacan que, dentro de las finanzas públicas de la educación, la medida de equidad utilizada más frecuentemente es el gasto por alumno. Éste se considera una proxy de la cantidad y calidad de la provisión del servicio.

Quienes sostienen que existe equidad educativa si se evidencia igualdad en el gasto por alumno se sitúan en la postura que defiende la igualdad en el acceso a la escolarización. Es decir, sustentan que un igual gasto por alumno implica igualdad en las oportunidades (Morduchowicz, 2003 Por el contrario, de evidenciarse una lógica de equidad en la asignación del gasto educativo en el sentido aquí propuesto, se deberían observar dos cuestiones al mismo tiempo: por un lado, una distribución de recursos económicos no igualitaria entre los distritos y, por el otro, que los distritos que reciban mayor financiamiento por alumno sean los menos aventajados. Si así fuera, se estaría avanzando hacia el objetivo de compensar las diferencias iniciales en busca de un resultado educativo común mínimo. Entonces, se calculó el coeficiente de GINI con el fin de describir el grado de desigualdad en el gasto por alumno entre los distritos de la provincia de Buenos Aires. La estimación se realizo tal como se detalló en la metodología y se utilizó como herramienta el software STATA 11.1.

Se obtuvo un valor del coeficiente de GINI igual a 0.37 para la variable Total de Fondos Provinciales en Infraestructura e Insumos por estudiante (TFPIIPA). Esto significa que hay desigualdad entre los distritos, ya que, cuando no se evidencia desigualdad, el coeficiente de GINI es cero. Como ya se ha explicado, la diferencia en la asignación de gasto promedio por alumno entre consejos escolares marca la existencia de individuos que reciben más que otros. Esta diferencia sería admitida, desde el punto de vista de equidad considerado, si los individuos que "más reciben" fueran los que "menos tienen". En término de distritos, esto significaría que aquellos que más fondos perciben son los que más necesidades socioeconómicas y educacionales poseen. Expresado de otro modo: de evidenciarse equidad en el sentido propuesto en este trabajo, los distritos con mayor proporción de establecimientos caracterizados como "desfavorables" y con mayores 
problemas de pobreza, analfabetismo y abandono escolar, deberían recibir una mayor cantidad de recursos por estudiante.

Dado que el cálculo del coeficiente de GINI no informa sobre lo descrito en el párrafo anterior, se amplió el análisis empírico haciendo uso de una regresión econométrica al igual que Ontiveros Jimenez (2003). Por ello, con base en los antecedentes expuestos y al marco teórico desarrollado, se planteó el siguiente modelo de Mínimos Cuadrados Ordinarios, representado por la ecuación (3):

$$
\mathrm{TFPII}_{\mathrm{i}}=B_{0}+B_{1}{ }^{*} \mathrm{FP}_{\mathrm{i}}+82 * \mathrm{M}_{\mathrm{i}}+B_{3}{ }^{*} \mathrm{ED}_{\mathrm{i}}+B_{4}{ }^{*} \mathrm{AIP}_{\mathrm{i}}+B_{5}{ }^{*} \mathrm{UEPE}_{\mathrm{i}}+B_{6}{ }^{*} \mathrm{TA}_{\mathrm{i}}+B_{7}{ }^{*} \mathrm{PP}_{\mathrm{i}}+B_{8}{ }^{*} \mathrm{NBI}_{\mathrm{i}}+B_{9}{ }^{*} \mathrm{~A}_{\mathrm{i}}+\mu_{\mathrm{i}}
$$

Donde TFPII $_{\mathrm{i}}$ es el financiamiento que recibe el concejo escolar del distrito i para hacer frente a los gastos de insumos y mantenimiento de infraestructura. $B_{0}$ es un término constante; el resto de las $B$ son los coeficientes que acompañan a las variables independientes descritas en la sección anterior y $\mu_{\mathrm{i}}$ es el término de error que muestra la diferencia residual entre los valores observados y estimados en cada distrito.

Se utilizó el comando reg para llevar a cabo la regresión en el software indicado (STATA 11.1) y se hizo la corrección por heteroscedasticidad utilizando el comando robust, provisto por el mismo software ${ }^{6}$. Los resultados obtenidos se detallan en las Tablas 1 y 2.

\section{Tabla 1.}

Características generales de la estimación

Variable dependiente: TFPII
Cantidad de observaciones $=114$
Prob $>\mathrm{F}=0,000$

$\mathrm{R}^{2}=0,8375$ (p-value de la prueba de significatividad conjunta)

Nota: Elaboración propia.

6 Se utiliza este comando que corrige heteroscedasticidad, es decir la violación al supuesto de varianza constante del término estocástico u. La corrección que lleva a cabo este comando es en términos generales y se elige utilizarlo porque, si bien el test "Breusch-Pagan / CookWeisberg test for heteroskedasticity" indico la presencia de heterosedasticidad, no se detectó una forma funcional específica de la misma, como para realizar una corrección particular. Es preciso corregir por heterosedasticidad porque de lo contrario, si bien los estimadores no presentarán sesgo, se verán afectadas las pruebas F y t, indispensables a la hora de sacar conclusiones (Douglas y Ramirez, 2011). Para mayores detalles sobre heterosedasticidad ver "Introducción a la econometría: Un enfoque moderno" (Wooldridge, 2001). 


\section{Tabla 2.}

Resultados de la estimación

\begin{tabular}{lll}
\hline Variables explicativas & Signo del coeficiente & P-value \\
\hline FP & 0,49 & 0,659 \\
MATRÍCULA (M) & $\mathbf{6 1 , 5 4}$ & $\mathbf{0 , 0 0 0}$ \\
ED & 5565,64 & 0,165 \\
AIP & 12416,59 & 0,220 \\
UEPE & $-663460,5$ & 0,373 \\
TA & $-10345,53$ & 0,669 \\
PP & $-151817,1$ & 0,278 \\
NBI & $-2229,58$ & 0,435 \\
ANALFABETISMO & $\mathbf{7 2 9 7 1 7 , 4}$ & $\mathbf{0 , 0 4 1}$ \\
CONSTANTE & 156253 & 0,896 \\
\hline
\end{tabular}

Nota: Elaboración propia.

Como se puede observar en la Tabla 1, se ha logrado un buen ajuste del modelo, ya que explica un $83,75 \%$ de la variabilidad en los fondos recibidos por cada distrito (ver valor del $\mathrm{R}^{2}$ ). Asimismo, el test $\mathrm{F}$ de significatividad conjunta refleja que se rechaza la hipótesis nula de que todos los coeficientes son iguales a cero $(p$-value $=0)$, por lo que se puede decir que el grupo de variables incorporadas al modelo explica, en forma simultánea, la variación del "Total de Fondos Provinciales en Infraestructura e Insumos educativos" que recibe cada distrito, a la vez que también refleja qué razones hay detrás de la forma en que se distribuye el total de fondos provinciales destinados a infraestructura e insumos para la educación.

En relación con los resultados obtenidos, cabe señalar que sólo hay dos variables estadísticamente significativas: "Matrícula" y "Analfabetismo" (ver la información en negrita y cursiva de la Tabla 2). La primera, es una variable que fue incorporada al modelo como control; mientras que la segunda fue incluida con el fin de corroborar la hipótesis.

El signo del coeficiente que acompaña la variable "Matrícula" es el esperado, es decir, positivo; a mayor cantidad de estudiantes por distrito, mayor es la cantidad de fondos asignados a éste. El valor de dicho coeficiente significa que, manteniendo el resto de los factores constantes, cada distrito recibe $\$ 61,54$ anuales adicionales por cada alumno matriculado. Este monto, que en principio puede parecer bajo, se vuelve relevante si se considera la gran cantidad de estudiantes que cursan en algunos distritos, lo que implica que el tamaño distrital tiene un peso relativo alto al momento de decidir cómo distribuir los fondos destinados a insumos e infraestructura educativos.

Por otra parte, el signo de la variable "Analfabetismo" también es positivo, lo cual demuestra cierto criterio de equidad en la distribución del gasto desde el gobierno central. 
Dicho de otro modo, cuanto mayor es el porcentaje de analfabetos en el distrito, mayor es la cantidad de fondos recibidos. Más precisamente, manteniendo el resto de los determinantes constante, la asignación de gasto educativo anual se incrementa en $\$ 729.717,4$ por cada punto porcentual de analfabetismo adicional. Esto refutaría la hipótesis propuesta, sin embargo, el resto de las variables que se incluyeron con el fin de probarla (ED, NBI, TA y AIP) resultaron no significativas estadísticamente, lo cual iría a favor de la hipótesis. Por lo tanto, de acuerdo con la muestra de datos utilizada, no se observa evidencia totalmente concluyente. De todas maneras, considerando que sólo una de cinco de las variables incluidas con el fin de corroborar la hipótesis resultó ser significativa estadísticamente, parece haber una mayor cantidad de empírea tendiente a confirmarla.

En suma, a partir del análisis empírico se observa que la única razón vinculada a la equidad que hace que un distrito reciba mayor financiamiento que otro tiene que ver con el grado de analfabetismo de su población; no se tienen en cuenta otras consideraciones esenciales como la proporción de estudiantes en los primeros niveles, el nivel de pobreza, la tasa de abandono y la proporción de establecimientos caracterizados como desfavorables. De este modo, puede concluirse que con base en los datos aquí estudiados la evidencia a favor de la hipótesis es mayor que la que la refuta. Por lo que se deduce que los criterios de equidad no son la guía rectora de la asignación del gasto educativo en la Provincia de Buenos Aires.

\section{Conclusiones}

En este estudio se analizó la distribución del gasto educativo en la provincia de Buenos Aires, Argentina. En función de la muestra de datos disponibles, los resultados del análisis empírico reflejan que es muy débil la evidencia a favor de la existencia de una lógica de equidad en la distribución de los recursos, en el sentido aquí propuesto. En principio, se calcula el valor del coeficiente de GINI para analizar el grado de desigualdad en la asignación de fondos entre los distritos de la provincia de Buenos Aires. Se observa que dicho valor es mayor a cero y a partir de allí se concluye que la distribución del gasto educativo no es igualitaria.

En segundo lugar, se estudia si dicha desigualdad es progresiva -en el sentido que sean los distritos menos aventajados los que reciban una mayor cantidad de dinero. De acuerdo con la metodología utilizada, se encontró que los recursos se reparten entre los diferentes distritos, mayoritariamente, en función de la matrícula, y que la evidencia a favor de la premisa de equidad expresada como: "deben recibir más los que menos tienen" es muy débil. Sólo una de las cinco variables incluidas en el modelo con el fin de probar la hipótesis resultó ser estadísticamente significativa. Específicamente, se halló que la distribución de los fondos depende en forma directa del grado de analfabetismo de los distritos, pero al mismo tiempo se encontró que la distribución de los fondos es independiente de la pobreza, de la tasa de abandono escolar, del porcentaje de matrícula en los niveles iniciales y de la proporción de establecimientos clasificados como desfavorables.

Lo explicado en los párrafos anteriores permite concluir que se confirma la hipótesis presentada en la introducción; es decir, que la distribución de los fondos a utilizar en gastos de infraestructura e insumos educativos no se rige, o lo hace muy débilmente, por el principio 
de equidad aquí propuesto. Entonces, las posibilidades de acceder, permanecer y transitar con éxito en el sistema educativo se muestran muy limitadas para aquellos estudiantes que pertenecen a los distritos que presentan mayores dificultades; ya que están recibiendo menos recursos que aquellos que se encuentran en una situación de ventaja. Esto, incluso, implica que puedan generarse círculos viciosos.

Finalmente, cabe señalar que es tarea de futuras investigaciones replicar el análisis realizado en la provincia de Buenos Aires tomando información correspondiente a otros períodos. De esta manera, será posible estudiar si la lógica de distribución hallada es o no una decisión política sistemática.

\section{Referencias}

Aghion P. y Howitt, P. (1998). Endogenous Growth Theory [Teoría del Crecimiento Endógeno]. Massachusetts: MIT Press.

Alas, M. y Moncada, G. (2010). Problemas de equidad en el sistema educativo hondureño. Revista Iberoamericana de Evaluación Educativa, 3(3), 135-151.

Baanante, M. J. y Geldres, I. A. (2003). La inversión pública en educación: proceso de asignación $y$ determinantes de la distribución del gasto por alumno. Lima, Perú: Instituto Apoyo.

Barro, R. J. y Lee, J. W. (2013). A new data set of educational attainment in the world, 1950 to 2010 [Nueva base de datos sobre rendimiento educativo en el mundo, desde 1950 hasta 2010], Journal of Development Economics, 104(C), 184-198. Doi: http://dx.doi. org/10.1016/j.jdeveco.2012.10.001

Birdsall, N. y Lodoño, J. (1997) Asset Inequality Matters: An Assessment of the World Bank's Approach to Poverty Reduction [La desigualdad patrimonial importa: Una evaluación del Enfoque del Banco Mundial para reducir la pobreza] The American Economic Review, 87 (2), 32-37.

Calero, J., Escardíbul, J. O. y Mediavilla, M. (2006). Notas para la construcción de un sistema de indicadores sobre desigualdad y educación en América latina. Boletín de SITEAL, 5. 4-52. Buenos Aires: IIPE-UNESCO. Recuperado de http://www.siteal.iipe-oei.org/sites/ default/files/boletin_05.pdf

Castello, A. y Doménech, R. (2008). Human capital inequality, life expectancy and economic growth [Inequidad en Capital Humano, esperanza de vida y crecimiento económico]. The Economic Journal, 118(528), 653-677. Doi:10.1111/j.1468-0297.2008.02136.x

Cohen, G. A. (1989). On the currency of egalitarian justice [Vigencia de la justicia igualitaria]. Ethics, 99(4), 906-944. Doi: http://dx.doi.org/10.1086/293126 
De la Torre, R. (2004). El Índice de Desarrollo Humano y la Asignación del Gasto Público por Entidad Federativa en México. Publicaciones IIDSES. México D.F: Instituto de Investigaciones sobre Desarrollo Sustentable y Equidad Social.

De Mello y Souza, A. (2006). Financiamiento de la educación en América Latina. Enseñanzas de la experiencia. En A. Morduchowicz (Ed.), Equidad y financiamiento de la educación en América Latina (pp. 19-57). Ciudad Autónoma de Buenos Aires, Argentina: IIPE-UNESCO.

Dirección Provincial de Información y Planeamiento Educativo de la Provincia de Buenos Aires (2012). Glosario. Recuperado de www.abc.gov.arlainstitucion/organismos/ planeamiento/infoestadistica/glosario.htm

Douglas C. y Ramirez V. (2012). Heterocedasticidad. Recuperado de www.webdelprofesor.ula.ve/ economia/dramirez/MICRO/FORMATO PDF/Materialeconometria/HETEROCEDASTICIDAD.pdf

Formichella, M. M. (2010). Educación y desarrollo: Análisis desde la perspectiva de la equidad educativa interna y del mercado laboral. (Tesis de doctorado). Departamento de Economía, Universidad Nacional del Sur, Bahía Blanca, Argentina.

Formichella, M. M. (2011). Análisis del concepto de equidad educativa a la luz del enfoque de las capacidades de Amartya Sen. Revista Educación35 (1),1-36. Doi: http://dx.doi. org/10.15517/revedu.v35i1.463

Gasparini L. (2002). On the measurement of unfairness: an application to high school attendance in Argentina [Sobre la medición de la injusticia: una aplicación a la asistencia en la escuela secundaria en Argentina]. Social Choice and Welfare, 19 (4), 795810. Doi: 10.1007/s003550200156

Gil Flores, J. (2014). Factores asociados a la brecha regional del rendimiento español en la evaluación PISA. Revista de Investigación Educativa, 32(2), 393-410. Doi: http://dx.doi. org/10.6018/rie.32.2.192441

Guadagni, A. (2007). Los próximos 25 años. Conferencia presentada en la XLII Reunión Anual de la Asociación Argentina de Economía Política (AAEP): Bahía Blanca, Argentina.

Honorable Cámara de diputados y senadores de la Provincia de Buenos Aires (2003). Ley Provincial $\mathrm{N}^{\circ}$ 13010. Recuperado de http://www.gob.gba.gov.ar/legislacion/ legislacion/l-13010.html

Jaramillo, M. y Artiaga, I. (2004). La inversión pública en educación: proceso de asignación y determinantes del gasto por alumno. Economía y sociedad, 54. 1-7. 
Llach, J. (2006). El desafío de la equidad educativa. Ciudad Autónoma de Buenos Aires, Argentina: Granica.

López, N. (2006). Equidad educativa y desigualdad social. Ciudad Autónoma de Buenos Aires, Argentina: IIPE-UNESCO.

López, R., Thomas, V. y Wang, Y. (1998). Addressing the Education Puzzle. The Distribution of Education and Economic Reforms [En dirección al enigma educativo: La distribución de la educación y las reformas económicas]. Policy Research Working Papers, 2031. Washington, D.C.: World Bank.

Lucas R. (1988). On the mechanics of development planning [Sobre los mecanismos de la planificación del desarrollo]. Journal of Monetary Economics. 22, 3-42. Doi: http://dx.doi. org/10.1016/0304-3932(88)90168-7

Lugo M. A (2005). Medidas de desigualdad para variables educativas. Boletín de SITEAL, 4. 1-47. Ciudad Autónoma de Buenos Aires: IIPE-UNESCO. Recuperado de http://www. siteal.iipe-oei.org/sites/default/files/boletin_04.pdf

Mezzadra, F. y Rivas, A. (2005). Coparticipación y equidad educativa: un debate pendiente en el campo de la educación. Publicaciones CIPPEC.Políticas Públicas. Análisis. 8. 1-5. Ciudad Autónoma de Buenos Aires: CIPPEC. Recuperado de http://nexos.cippec.org/ documentos/politicas_publicas_a.pdf

Molina Morales, A., Amate, I. y Guarnido, A. (2011). El gasto público en educación en los países de la OCDE: condicionantes económicos e institucionales. eXtoikos, 4, 37-45. Recuperado de http://www.extoikos.es/n4/pdf/7.pdf

Morduchowicz, A. (2003). Discusiones de economía de la educación. Ciudad Autónoma de Buenos Aires, Argentina: Losada.

Neyra Aráoz, G. (2010). Determinación de las necesidades de gasto fiscal en el nivel regional. Consorcio de Investigación Económica y Social (CIES). Recuperado de http://www.cies. org.pe/sites/default/files/investigaciones/determinacion_de_las_necesidades_de_gasto_ fiscal_en_el_nivel_regional.pdf

Nina, O., Molina, O., Barrientos, P. y Aguilar, P. (2006). ¿Es equitativa en Bolivia la asignación del gasto público en educación? Revista Latinoamericana de Desarrollo Económico-rdle, 7, 57-96.

Ontiveros Jiménez, M. (2001). Gasto educativo y políticas distributivas de la educación primaria en México. Revista Latinoamericana de Estudios Educativos, XXXI (003), 53-77. 
Ontiveros Jiménez, M. (2003). Gasto Público y provisión de servicios. El caso de la educación primaria en México. El Trimestre Económico, 70 (279), 579-611.

Ribotta, S. (2006). Las desigualdades económicas. Un estudio desde el igualitarismo contemporáneo. (Tesis de Doctorado). Universidad Carlos III, Madrid, España.

Roemer, J. E (1995). Equality and responsibility. Boston Review Forum, Social Equality and Personal Responsibility. 20 (2), 3-7.

Rojas, M. y Formichella, M. M. (2011). Cambios en el Sistema Educativo Argentino: El proceso de descentralización y la posterior reforma. Madrid:Editorial Académica Española.

Sanchez, F. (2006). Descentralización y progreso en el acceso a los servicios sociales de educación, salud y agua y alcantarillado. Documento CEDE 2006-15. Recuperado de http://economia.uniandes.edu.co/investigaciones_y_publicaciones/CEDE/Publicaciones/ documentos_cede/2006/descentralizacion_y_progreso_en_el_acceso_a_los_servicios_ sociales_de_educacion_salud_y_agua_y_alcantarillado .

Santos M. (2007). Un modelo de trampa de pobreza con capital humano y calidad de la educación. Ponencia presentada en la XLII Reunión Anual de la Asociación Argentina de Economía Política (AAEP). Bahía Blanca, Argentina.

Sen A. (1999). Desarrollo y Libertad. México, D. F: Ed. Planeta.

Sen, A. (1979). Equality of what? The tanner lecture of human values. [¿Equidad de qué? Una lectura comparativa de los valores humanos]. Recuperado de http://sjmse-library. sch.ng/E-Books\%20Phil/Sen\%20Equaliy\%20of\%20what.pdf

SEN, A. (1997) La desigualdad económica. Madrid, España: Fondo de Cultura Económica.

Tedesco, J. C. (2000). Educar en la sociedad del conocimiento. México, D. F.: Fondo de Cultura Económica.

Thomas, V. Wang, Y. y Fan, X. (2001) Measuring education inequality: Gini Coefficients of Education [Midiendo la inequidad de la educación: Los coeficientes Gini de la educación]. Policy research working papers 2525. Washington, D.C.: World Bank.

Thomas, V. Wang, Y. y Fan, X. (2002). A new dataset on inequality in Education: Gini and Theil indices of schooling for 140 countries, 1960-2000 [Una nueva base de datos sobre Inequidad en Educación: Coeficientes de GINI y Theil de escuelas de 140 países, 1960- 
2000]. Recuperado de http://www.researchgate.net/publication/252435375_A_New_ Dataset_on_Inequality_in_Education_Gini_and_Theil_Indices_of_Schooling_for_140_ Countries_1960-2000

Wooldridge, J. (2001). Introducción a la econometría: Un enfoque moderno. México, D. F: Paraninfo. 